\title{
A Small-Scale, Contactless, Pure Bending Device for In-situ Testing
}

\author{
J.P.M. Hoefnagels ${ }^{1}$ - A.P. Ruybalid ${ }^{1}$ - C.A. Buizer ${ }^{1}$
}

Received: 18 February 2015 / Accepted: 25 May 2015 / Published online: 23 June 2015

(C) The Author(s) 2015. This article is published with open access at Springerlink.com

\begin{abstract}
The introduction of flexible electronics calls for new ways of measuring the mechanical limits and failure mechanisms resulting from (large) bending loads during manufacturing and application. Therefore, an autonomous, miniaturized, pure bending test apparatus was developed to investigate material systems at the microscopic level. Improvements to conventional bending test methods are: (1) well-defined pure moment application without friction or local pressure contacts, through active feedback control of parasitic axial and transverse forces at the specimen edges, (2) continuous reversibility of the loading direction within an angular range of $-114^{\circ} \leq \theta \leq 114^{\circ}$ (minimum radius of $2 \mathrm{~mm}$ ), (3) no view-obstruction from both the thickness and in-plane perspective, enabling in-situ optical and scanning electron microscopic studies of failure mechanisms under constant field-of-view. The pure bending test setup was validated by moment-curvature measurements of monocrystalline silicon. The setup's continuous load-reversibility and strain determination were validated by cyclic tests and in-situ digital image correlation, respectively, on polyethylene napthalate specimens. Furthermore, in-situ microscopic failure analysis was demonstrated on
\end{abstract}

J.P.M. Hoefnagels

j.p.m.hoefnagels@tue.nl

\author{
A.P. Ruybalid \\ a.p.ruybalid@tue.nl \\ C.A. Buizer \\ caspar.buizer@gea.com
}

1 Department of Mechanical Engineering, Eindhoven University of Technology, P.O. Box 513, 5600 MB Eindhoven, The Netherlands multilayered flexible electronics, revealing fracture, delamination and buckling.

Keywords Pure bending - Small-scale mechanical testing $\cdot$ Integrated testing $\cdot$ In-situ scanning electron microscopy $\cdot$ Flexible electronics

\section{Introduction}

Many modern electronic applications, including solar cells, light emitting diodes, and displays, make use of thin film technology. In such applications, functional components are stacked onto a thicker substrate, which provides mechanical support to the entire material system. Conventionally, the substrate material is made of a rigid ceramic (e.g., single crystal silicon). However, improved compliance of the substrate material is attractive, since it extends the design freedom to realize, e.g., curved geometries and bendable or flexible applications, which are lightweight and less susceptible to structural failure than their rigid equivalents. Another important advantage is the potential ability to produce flexible electronic systems in a roll-to-roll process [1], which is more efficient and cost-effective compared to standard silicon-based processing techniques.

Thus far, successful realization of flexible electronic devices is hindered by persistent problems occurring during production and application, which mainly concern mechanical reliability of the used material systems. Substrates are typically made of polyethylene naphtalate (PEN) or polyethylene terephthalate (PET), and have thicknesses in the order of $100 \mu \mathrm{m}$, while functional layers (e.g., organic light-emitting materials) have thicknesses ranging from a few hundreds of nanometers up to a few micrometers. Besides the substrate and functional layers, flexible 
electronics require other materials that provide (1) protection to the flexible substrate against hostile chemical processing steps, (2) mechanical protection against wear or scratching, (3) permeation barriers to prevent oxidation and hydroxidation of electrodes and functional materials, (4) semi-transparent electrodes in, e.g., light-emitting or light-capturing systems $[1,2]$. Unfortunately, the thin film materials that possess these qualities are typically brittle, and therefore jeopardize the required flexibility of the device. Risk factors for failure include: (1) residual stress in thin films after certain production processes, due to thermal and elastic mismatch between different material layers [1, 3-8], (2) flaws in the material, caused by manufacturing processes [6], and (3) excessive loading during production (e.g., in the roll-to-roll process) leading to deformations exceeding critical limits of (brittle) material layers [4, 9]. Important failure modes in these systems are (1) brittle fracturing of thin barrier coatings, (2) interface delamination of adjacent layers, (3) buckling of more compliant materials, and (4) fatigue failure during cyclic loading.

To improve the production processes and material systems of flexible electronics, mechanical testing is required in combination with microscopic investigatory techniques to observe the phenomena that are responsible for failure of the device. In combination with numerical simulation, this facilitates quantification of, e.g., stresses and strains within material layers upon (microscopic) failure, making identification of bulk and adhesion properties of materials and interfaces possible. In turn, this allows for bettering the understanding of significant failure modes, and the realization of predictive models.

Since bending is the prominent loading mode in the roll-to-roll process and applications of flexible electronic devices, a bending test is the most suitable method for investigating mechanical behavior of flexible electronic devices. Conventional bending methods include 2-point, 3-point, and 4-point bending tests, which all make use of local pressure contact to induce bending to the specimen. At these points of contact, the loading conditions throughout the bent specimen are not trivially determined, due to friction and local deformation (possibly inducing plasticity or damage in certain material layers) [10]. This is a major disadvantage if critical failure values are wished to be quantified, and material or interface properties need to be measured. Because the true boundary conditions, applied during experimentation, are not completely known, accurate simulation of experiments becomes unfeasible, jeopardizing the reliability of predictive models. There are also practical limitations that make conventional bending methods unsuitable for investigating microscopic failure behavior in flexible electronics. For example, due to the use of view-obstructing contact points, not all areas of the tested specimen can be observed during microscopic investigation. The same pressure contacts restrict the possibility to perform cyclic tests, since it is difficult to reverse the loading direction in a continuous manner, as occurs in reality during a roll-to-roll process, and which is needed to examine elastic spring-back and Bausschinger effects. Furthermore, large deformations, as are of importance in flexible electronics, are difficult to realize when using pressure contact-based test setups.

To overcome these problems, a new testing method was developed that solely applies a bending moment to the edges of a specimen without inducing transverse or axial forces that would cause so-called parasitic moments. This imposes the well-defined state of pure bending [11], which, for a homogeneous material, causes a straight specimen to be deformed toward a perfect circular arc, while the moment is constant along the specimen. In addition, for a homogeneous specimen of isotropic or orthotropic elastic material under pure bending (with an assumed plane stress condition in thickness direction and either a plane stress or a plane strain condition in width direction), the complete stress and strain tensors can be derived analytically in terms of the material parameters, the bending radius $R_{0}$, and the distance from the neutral surface $r-R_{0}$, giving a simple expression for the axial strain:

$\varepsilon_{t t}=\frac{r-R_{0}}{R_{0}}$.

The contactless, frictionless, pure bending test setup has been optimized for testing thin specimens of flexible electronic systems in combination with optical and scanning electron microscopic techniques. Even for inhomogeneous multilayer specimens exhibiting complex material behavior, the moment acting on the specimen's cross section is kept constant along the specimen length, and any parasitic moments are eliminated by the setup. Because of the welldefined loading conditions in the specimen, experiments can be compared well with simulations.

This paper describes the design criteria and design of the developed testing device in "Design Considerations and Criteria" and "Design Implementation", respectively. In "Setup-Validation Measurements", validation measurements are shown, which verify that the new testing device meets the design criteria. Finally, the setup's performance is demonstrated on different test-case examples in "Demonstration of Setup Potential", which is followed by the "Conclusions and Recommendations".

\section{Design Considerations and Criteria}

The most important requirement for the bending setup is that only a pure bending moment is applied to the specimen. 
This leads to the design criterion that all parasitic moments, caused by forces applied at the clamps, should be negligible compared to the applied pure moment and that these forces should be measured and eliminated by the setup. With these requirements in mind, existing bending setups can be investigated.

To overcome the above-mentioned complications associated with conventional (2-point, 3-point, 4-point) bending methods, Yoshida et al. [12] developed a large-amplitude, cyclic, bending setup with the possibility to apply reversed loading. Yoshida's setup uses a contactless principle to induce bending by relative rotations of two clamps that are mounted on sliders. Although elegant in design, friction of the sliders induces parasitic loads to the bent sample, causing the state of pure bending to be lost. Moreover, friction introduces hysteresis in cyclic tests which is nontrivial to calibrate out, and the influence of friction increases when the setup would be miniaturized to enable in-situ microscopic observation [13]. Therefore, Boers et al. [10] replaced Yoshida's sliders for frictionless air bearings, enabling their setup to perform large amplitude, cyclic, pure bending in a contactless and frictionless fashion. Boers et al. also argued that, in general, it is not possible to include the influences of contact and friction in numerical tests. This leads to the design criterion stating that the effects of contact and friction should be negligible compared to the applied pure moment in order to enable realistic comparison with the numerical tests.

Wiklund et al. [14], Frei et al. [15], and Buchanan et al. [16] already performed in-situ, 4-point bending tests in a scanning electron microscope (SEM), which enabled them to examine crack propagation phenomena at the microscopic level. For the here-presented bending setup, insitu SEM observation of pure bending failure is also an important requirement, as it will greatly enhance the possibilities to unravel the underlying physical origin of the bending failure. In-situ SEM characterization of mechanical behavior of materials at the microscopic scale requires miniaturization of the testing device and adaptation to additional constraints, such as vacuum compatibility of all setup components, an increased influence of friction, and a horizontal working plane for the vertical SEM viewing direction. Sufficiently high load application can be challenging due to the limited space in a SEM vacuum chamber (e.g., $260 \times 225 \times 90 \mathrm{~mm}^{3}$ for a FEI Quanta 600 SEM) [17], while the mitigation of friction can trigger unconventional design choices, such as electron discharge machined elastic hinges [13]. Ideally, the contactless, frictionless pure bending setup for large amplitude, cyclic loading, developed by Boers et al., should simply be miniaturized. Realizing this is, however, not trivial, because the air bearings that were used by Boers et al. cannot be adopted, since the setup should be able to operate in vacuum circumstances. Furthermore, magnetic fields may affect the electron beam in the SEM, causing a disturbance during visualization and should therefore be avoided. Moreover, when focusing the electron beam on a certain region of interest, the specimen should remain inside a $100 \times 100 \mu \mathrm{m}^{2}$ field-of-view, which forms a challenging requirement.

Ideally, the setup should be able to bend flexible electronic specimens to failure in order to study the corresponding mechanisms, such as interface delamination, buckling, and fracture. A detailed analysis considering various specifications of flexible electronics, including the fracture strain, other material properties of commonly used materials (polymers, metals, and ceramics), and the range of typical layer and specimen thicknesses yield the design requirements of an applicable bending radius of minimally $2 \mathrm{~mm}$ and a bending moment up to $0.06 \mathrm{Nm}$ to be able to test relevant systems. Note that, although the pure bending setup is developed for flexible thin films, the setup should also be applicable for testing other materials like metal foils, laminar composites and paper. Consequently, quick and easy modifications should be allowed to make this possible. The setup should also be usable for a range of sample dimensions. Additionally, the setup should enable the investigation of time-dependent mechanical behavior, such as relaxation and creep, requiring the ability to perform autonomous tests under moment control or angular control. Finally, it is most important that the testing conditions are well-defined and reproducible. These considerations result in the following design criteria:

- pure moment application along the entire specimen, in a contactless and frictionless manner

- well-defined and reproducible testing conditions, with autonomous operation

- range of specimen sizes: $4 \times(0-3) \times(0-0.4) \mathrm{mm}^{3}$

- minimum bending radius of $2 \mathrm{~mm}$ and a bending moment up to $0.06 \mathrm{Nm}$

- allow for large amplitude, reversed (and cyclic) loading

- allow for creep and relaxation tests

- compact, vacuum compatible design with a horizontal working plane for in-situ experiments with SEM (260 $\times 225 \times 90 \mathrm{~mm}^{3}$ volume of a FEI Quanta 600 sample chamber).

- region of interest should remain inside a $100 \times$ $100 \mu \mathrm{m}^{2}$ field-of-view

\section{Design Implementation}

The proposed design of the bending setup is based on the analytic description of the kinematics of a homogeneous, 
elastic, purely bent beam. Note, however, that by ensuring the application of a pure moment without other forces, the proposes principle also holds up for inhomogeneous specimens. A pure moment is imposed by prescribing motion paths of the clamped ends of the beam with the aid of a combination of displacement- and force-driven manipulators and load measurement systems. The underlying concept and eventual design are introduced in the following section.

\section{Conceptual Design}

Let us first consider a uniform specimen that behaves in an either orthotropic or isotropic, elastic manner over its full length. The conceptual design of the setup is based on analytically determined prescriptions of the clamps' relative motion that realizes a state of pure bending in such specimens.

The specimen with length $L_{0}$ in Fig. 1 is fixed in space at point $O$, which is the origin of a Cartesian coordinate system and represents the region of interest that will be examined by microscopic techniques (e.g., SEM). Only a one dimensional specimen is considered for now, i.e., the beam specimen has no width and thickness dimensions, meaning that the length of the specimen remains constant during bending. As a result of pure bending, the homogeneous beam curves according to a perfect circular arc. The coordinates $\left(x_{1}, y_{1}\right)$ and $\left(x_{2}, y_{2}\right)$ represent the location of the right and left clamped beam-ends with respect to fixed point $O$, and are formulated as follows:

$x_{1}=-x_{2}=\frac{L_{0}}{\theta} \sin \left(\frac{\theta}{2}\right)$,

$y_{1}=y_{2}=\frac{L_{0}}{\theta}\left(1-\cos \left(\frac{\theta}{2}\right)\right)$,

where the bending angle is represented by $\theta$. To allow for true pure bending, a model is proposed that uses two endplates tangentially connected to the specimen, which are schematically drawn by the thicker lines in Fig. 1, in which $\left(x_{r 1}, y_{r 1}\right)$ and $\left(x_{r 2}, y_{r 2}\right)$ are the coordinates of the rotation

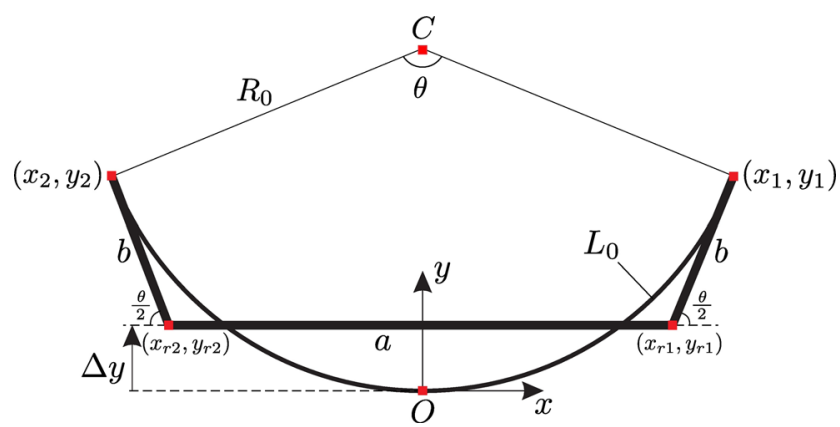

Fig. 1 Model to describe the kinematics of a homogeneous beam being bent by end-plates connected tangentially to the beam points, where the frame of reference is chosen such that the region of interest in the middle of the specimen remains fixed at the origin $O$. The clamped beam-end positions $\left(x_{1}\right.$, $\left.y_{1}\right)$ and $\left(x_{2}, y_{2}\right)$ of equations (2) and (3) can be equated to:

$x_{1}=-x_{2}=\frac{a}{2}+b \cos \left(\frac{\theta}{2}\right)$,

$y_{1}=y_{2}=\Delta y+b \sin \left(\frac{\theta}{2}\right)$,

which leads to the following system of equations for pure bending, written in matrix form as:

$$
\left[\begin{array}{lll}
\frac{1}{2} & 0 & \cos \left(\frac{\theta}{2}\right) \\
0 & 1 & \sin \left(\frac{\theta}{2}\right)
\end{array}\right]\left[\begin{array}{l}
a \\
\Delta y \\
b
\end{array}\right]=\left[\begin{array}{l}
\frac{L_{0}}{\theta} \sin \left(\frac{\theta}{2}\right) \\
\frac{L_{0}}{\theta}\left(1-\cos \left(\frac{\theta}{2}\right)\right)
\end{array}\right] .
$$

In order to adequately describe the motion of the clamps, restrictions to the degrees of motion-freedom of the rotation points must be applied. In total, eight different cases that provide restriction, can be considered. However, only three of those cases are suitable for solving the pure bending equations (equation (6)); (1) the case in which solely $y$-motion of the rotation points; $\Delta y$, and variation of distance $b$ are allowed, (2) the case where solely $x$-motion of the rotation points and variation of distance $b$ are allowed, and (3) the case in which both $x$ - and $y$-motion of the rotation points, but no variation of distance $b$ are allowed. The system is over- or under-constrained in all other cases. Through a detailed study of case (3), which requires the use of two linear actuators and one rotational stage for each clamp, it was concluded that the specimen chamber of the SEM is too limited in size for realization of this proposal, so it is discarded. Case (2) requires two actuators to move both rotation points synchronously, in opposite $x$-direction for the realization of symmetry, while case (1) only requires one actuator for $y$-motion. Consequently, case (1) forms the starting point for the design of the pure bending stage.

It is noted that several alternative strategies to prescribe clamp motion were investigated before reaching the abovedescribed conceptual design, but were found to contain limitations, as described by Hoefnagels [18].

\section{Setup Design}

The following subsection describes how the proposed conceptual design is realized in the setup. Because the specimens are small, the two rotation points would be forced to be located closely together, which would cause inconveniences in the construction. Therefore, the fixed distance $a$ between the rotation points is chosen to be zero, resulting in one single rotation hinge, instead of two, which reduces 
the complexity and costs of the design. When $a=0$ is postulated in equation (6), expressions for $b$ and $y$ become:

$b=\frac{L_{0}}{\theta} \tan \left(\frac{\theta}{2}\right)$,

$\Delta y=\frac{L_{0}}{\theta}\left(\frac{-1+\cos \left(\frac{\theta}{2}\right)}{\cos \left(\frac{\theta}{2}\right)}\right)$.

The setup, schematically shown in Fig. 2(a), consists of two individual clamps $c_{1}$ and $c_{2}$, which are attached to two bars of length $L_{1}$ that can rotate around the single fixed joint $J_{1}$. By using two push-pull rods of length $L_{2}$ that are linearly actuated at joint $J_{4}$, synchronous rotation of the bars with joints at $J_{2}$ and $J_{3}$, is accomplished. The linear actuator realizes rotation of the clamps by the translation $\Delta J_{4}$ of joint $J_{4}$, and is referred to as the rotation stage. Distance $b$ between the clamps and the fixed joint $J_{1}$ equals half the specimen length in zero-bending-angle condition, and is the same distance as earlier referred to in Fig. 1.

Equation (7) shows that distance $b$ should be varied to a specific value for each bending angle, i.e., follow a specific path, in order to realize a state of pure bending without any axial and transverse forces to cause parasitic moments in the specimen. The clamps should therefore move along the longitudinal direction of the bars. Mechanical bearings should not be used for this clamp movement since friction in the bearings cause parasitic loading of the sample. Air bearings are also not an option, because the setup will be used in (low) vacuum circumstances during in-situ SEM testing. Magnetic levitation cannot be used, because the strong magnetic fields would disturb the electron beam of the SEM and thereby the visualization. Free movement of the clamps by attaching them to low-stiffness leaf springs causes problematic dynamic behavior. Nevertheless, a solution can be found in the use of simple linear actuators.

Two linear actuators will move the clamps synchronously in the axial direction of the specimen with a magnitude $\Delta b$. In addition, one linear actuator realizes transverse motion $\Delta c$ of one of the two clamps to correct for asymmetry in the specimen, due to, e.g., inhomogeneities in the specimen. We accept the fact that symmetry of the setup is lost, because only small transverse motion is expected (i.e., minor asymmetry in the sample). The setup would be underconstrained if symmetry is realized by adding two transverse correction actuators instead of one. Furthermore, the entire setup is positioned on top of another linear actuator to keep the microscopic region of interest within the fieldof-view. The motion of this field-of-view stage represents the $\Delta y$-translation as described by equation (8). Figure 2(b) highlights the linear clamp actuators and the mechanism that keeps the region of interest within the field-of-view.

\section{Moment Measurement}

To quantify the applied loading during an experiment, the moment is measured by a frictionless principle in order to prevent application of additional forces that would produce undesired, parasitic moments. A moment sensor is realized by measuring a rotation $\phi$, and using a torsion spring with a low stiffness $k_{\phi}$ to effectively measure a moment, i.e., $M=k_{\phi} \phi$. This is done by a spring mechanism consisting of two serial elastic hinges, whose center-lines cross each other precisely in the specimen center-line at the clamp tip, which forms the virtual pivot point $P$ around which the system has an effective low torsion stiffness $k_{\phi}$. The concept is shown in Fig. 3(a). Rotation at this point causes a nearly linear, tangential displacement $\Delta x$ of an appendix of the clamp, which is measured by a contacless capacitive sensor (Lion precision D-C5), which is best suited in this case when
Fig. 2 conceptual design of the pure bending test setup, which uses two clamps $c_{1}$ and $c_{2}$ that grip the specimen and rotate around the single rotation point $J_{1}$. The clamps can also translate by $\Delta b$ and $\Delta c$ to compensate for axial and transverse forces

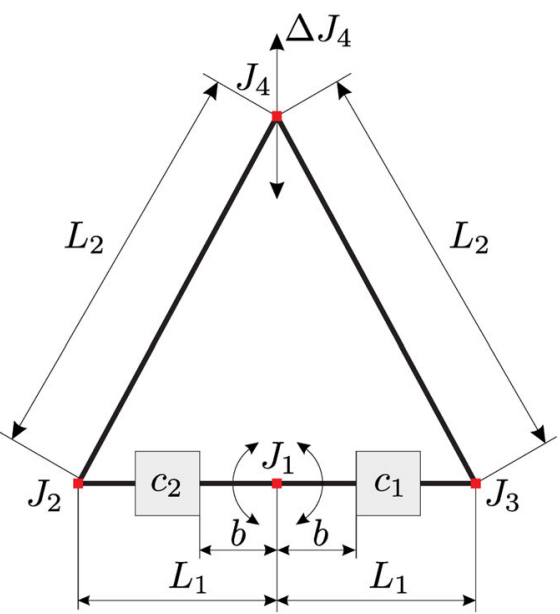

(a) rotation mechanism of the clamps

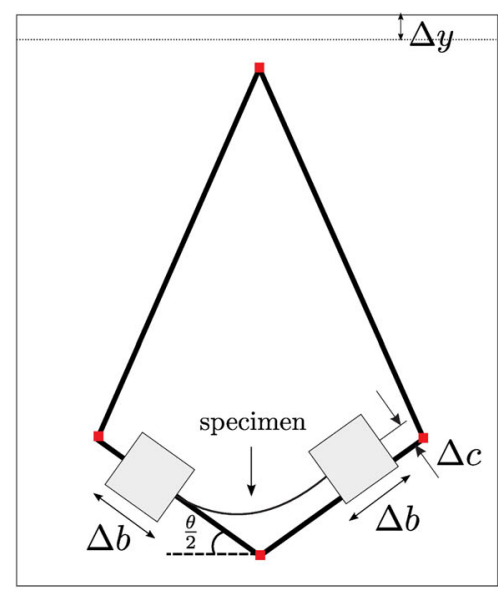

(b) linear translation mechanisms 
Fig. 3 the moment at virtual pivot point $P$, which coincides with the tip of the clamp at which the specimen is gripped, is effectively measured through four torsion springs and translation $\Delta x$ of a clamp-appendix by a capacitive sensor

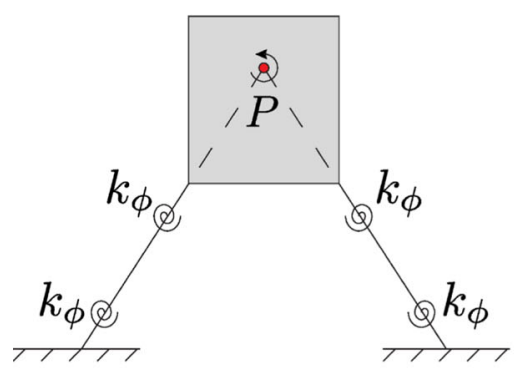

(a) conceptual design

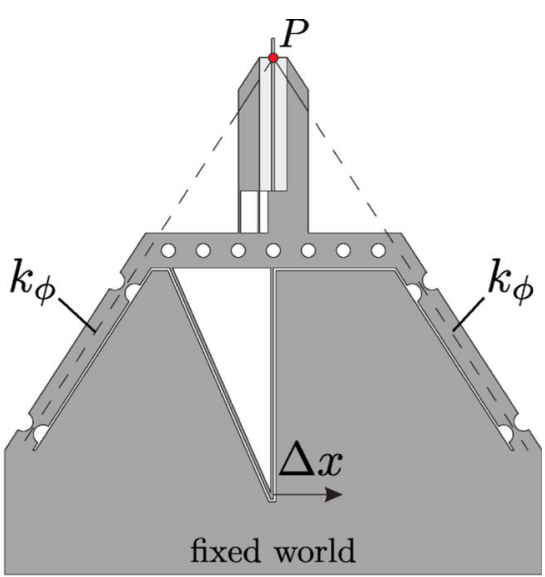

(b) actual design considering resolution versus range. The moment measurement system, embedded in one of the two clamps, as shown in Fig. 3(b), is designed such that the rotation by which the moment is measured is maximally $\phi_{\max }=0.024^{\circ}$, which is negligibly small compared to the large bending angle range of the setup (up to $114^{\circ}$ ). Therefore, the moment measurement does not significantly affect the applied bending angle during experimentation. Furthermore, the resolution of the realized moment sensor is $M_{\text {res }}=1.4 \mu \mathrm{Nm}$, which is governed by the resolution of the capacitive sensor that can measure displacements of $10 \mathrm{~nm}$, and the stiffness of the elastic hinges: $k_{\phi}=144\left[\frac{\mathrm{Nmm}}{\mathrm{mm}}\right]$. It is noted that the measurement system can easily be adapted by changing the stiffness of the elastic hinges so that other moment ranges can be measured if necessary (e.g., for other materials than flexible electronics).

\section{Force Feedback Control}

Material damage, plastic deformation, material defects, variation in geometric properties have significant effects on the deformation of a specimen and cause deviation from the perfect pure bending condition. Correction of clamp positioning must be made to realize sole moment-application, without any axial and transverse forces, in cases of heterogeneity of specimens and/or inelastic material behavior. Sensitive measurement of such undesired forces, that lead to parasitic moments, can be used for active feedback control of actuators to adjust the clamp positions. Therefore, very small axial and transverse force loads are allowed to act on the specimen, but should be negligibly small in comparison to the bending moment that is applied to the specimen. Moreover, the specimen should move freely, so the loads should be measured according to a contactless and hence frictionless principle.
Figure 4 schematically illustrates this concept for the case of elimination of the axial force in the specimen. A clamp grips the specimen and is attached to a base plate by two leaf springs that have a low, known stiffness in the direction in which the force in the specimen is to be sensed (axial or transverse). When deviation from pure bending occurs, the specimen will push or pull the clamp and load the leaf springs with a force $F$. The resulting displacement of the clamp is measured by a sensitive Linear Variable Differential Transformer (LVDT), which was found to be best suited for this purpose when considering resolution versus range. Initially, the distance between the LVDT and the clamp is $x_{0}$, but when a force acts on the specimen, the clamp displaces $\Delta x$ with respect to the base plate, through elastic deformation of the leaf springs. The displacement measurement is used to compensate the position of the base plate by an actuator to eliminate the force. This mechanism is also applied in transverse direction to eliminate the corresponding forces in the specimen. The force measurement system is embedded in one of the two clamps (since the other clamp measures the bending moment), of which an illustration is shown in Fig. 4(d). Two LVDTs (Macro Sensors CD 375025) measure the small translations $\Delta x_{\mathrm{AF}}$ and $\Delta x_{\mathrm{TF}}$ of the clamp with respect to the base plate that result from axial and transverse forces, respectively, acting on the specimen.

The strict design rule used to determine the allowable axial and transverse forces comes from the resolution of the moment sensor $M_{\text {res }}$ :

$F_{\text {lim }}=\frac{2 M_{\text {res }}}{L_{0}}=0.72[\mathrm{mN}]$,

which means that the forces may not cause parasitic moments that affect the moment measurement. The actuators that correct the clamp position to eliminate the forces have a minimum incremental motion step of $\Delta x_{\text {step }}=$ 
Fig. 4 illustration of the active feedback control system, with (a) the initial condition when no force acts on the specimen, (b) the displacement of the clamp with respect to the base plate due to an axial force $F$, and (c) the condition after the actuator has translated the base plate by $\Delta x$ to compensate for the force. The measurement system is embedded in one of the two clamps (d), which grips a specimen at point $A$

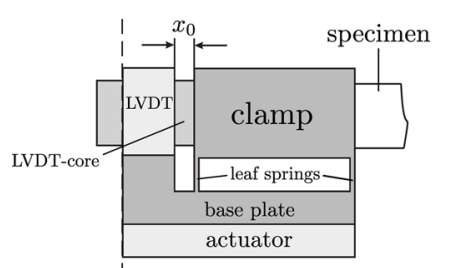

(a)

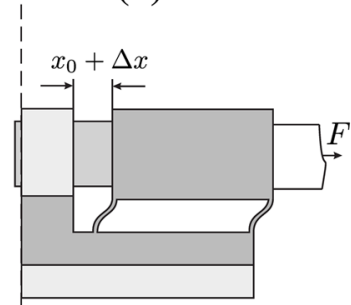

(b)

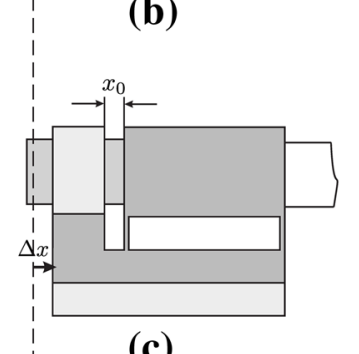

(c)

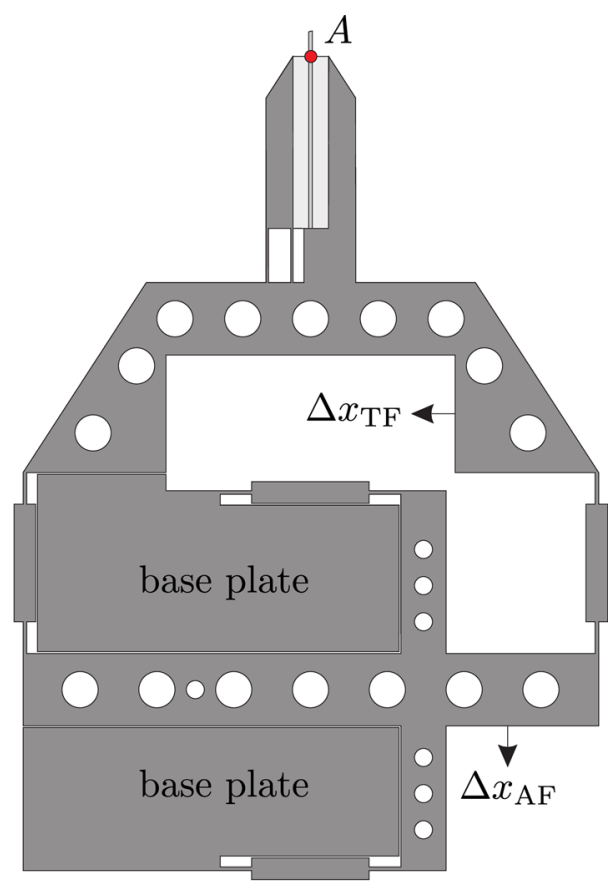

(d)
$0.3 \mu \mathrm{m}$. This information is used to determine the required stiffness of the leaf springs mechanisms in the clamp, which should at least be smaller than:

$k_{\mathrm{AF}}=k_{\mathrm{TF}} \leq \frac{F_{\text {lim }}}{\Delta x_{\text {step }}}=2600\left[\frac{\mathrm{N}}{\mathrm{m}}\right]$.

After realization of the setup, the stiffness of the leaf springs were experimentally measured to have values of $k_{\mathrm{AF}}=$ $171\left[\frac{\mathrm{N}}{\mathrm{m}}\right]$ and $k_{\mathrm{TF}}=164\left[\frac{\mathrm{N}}{\mathrm{m}}\right]$, so that unwanted forces that are well below the allowed limit of equation (9) can be measured and eliminated.

Although the force is measured in one of the two clamps, both clamps will be translated by linear actuators synchronously to compensate for the axial force, as was already mentioned in "Setup Design". Only one clamp will translate to compensate for transverse forces.

\section{Setup Realization}

The bending stage has a horizontal working plane, meaning that the specimen is bent around the vertical axis, and it can operate autonomously by following a prescribed angular-controlled or moment-controlled motion path. A CAD model and a photograph of the realized setup are shown in Fig. 5, in which the main components are indicated. The rotation stage is a linear actuator (Newport LTA-HS) that translates joint $J_{4}$ by $\Delta J_{4}$, and which has a minimum incremental motion of $0.1 \mu \mathrm{m}$. This translation is transformed into a rotation around fixed joint $J_{1}$ (minimal incremental rotation of $0.1 \mu \mathrm{rad}$ ), through two push-pull rods connecting joint $J_{4}$ to the joints $J_{2}$ and $J_{3}$. The moment and force measurement systems, discussed in "Moment Measurement" and "Force Feedback Control", are mounted on top of linear actuators (PI M-663) that compensate for axial and transverse forces in the specimen. As mentioned previously, both clamps have linear actuators that can translate in the axial direction of the specimen (synchronously) to eliminate the corresponding axial force. Only one of the two clamps, however, compensates for the transverse force by containing an additional actuator, stacked on top of the other, which can translate in the transverse direction (with respect to the specimen). The latter is the clamp on the right-hand side of the CAD model, which comprises the force measurement mechanism. Furthermore, the linear field-of-view actuator (Newport TRA-6PPV6) ensures that the specimen remains within a $100 \times 100 \mu \mathrm{m}^{2}$ area during bending, by translating the entire rotation stage. The whole setup is mounted on top of a base plate, which can be horizontally leveled by manual tilt-manipulators.

The clamping grippers, of which a closeup is shown in Fig. 5(b), are narrowed towards their tips, so that they can realize the minimum bending radius of $2 \mathrm{~mm}$ without touching each other. The red bars, seen in the picture, can be tightened by a screw to clinch the grippers, which are 


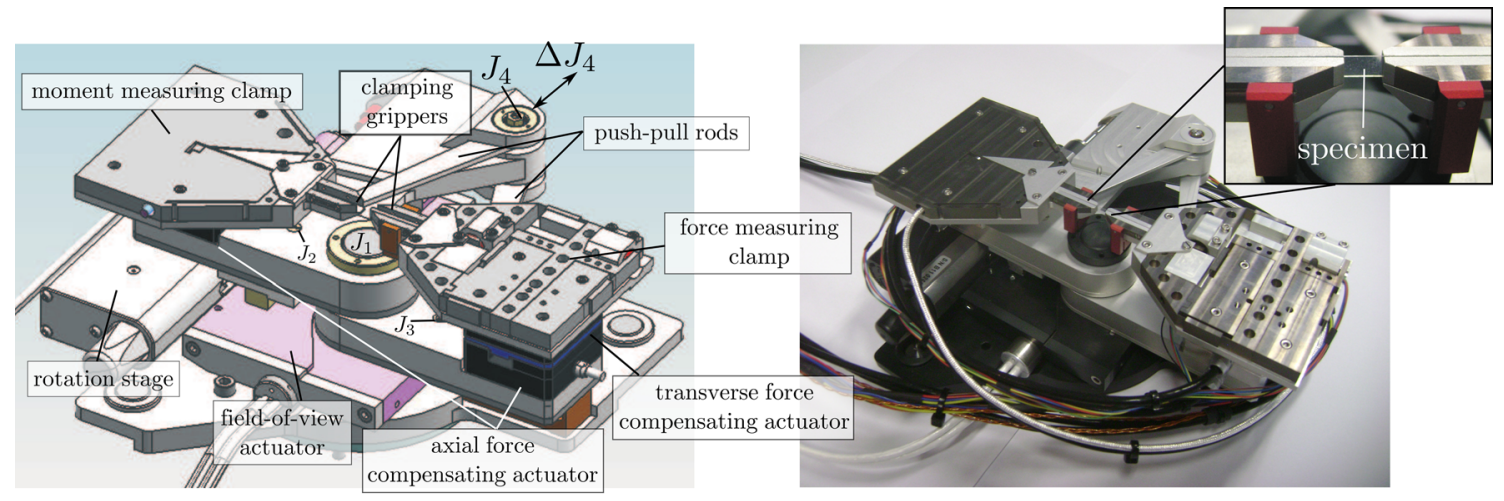

(a) CAD model

(b) photograph of the setup

Fig. 5 CAD model (a) and photograph (b) of the realized bending test setup. The joints $J_{1-4}$, as illustrated in the conceptual design of Fig. 2, are indicated in (a)

connected to the clamp body by elastic hinges, and securely clamp the specimen at the gripper-tips.

To allow for microscopic investigation of failure mechanisms during bending of a specimen, a right-angle mirror is used to realize both thickness and in-plane observation by optical microscopy, which is shown in Fig. 6. When observing the thickness dimension of a multilayered specimen, interface failure behavior can be studied, while the in-plane perspective allows for investigating in-plane fracture phenomena. Examples of such failure mechanisms observed by different microscopic techniques in combination with the bending test setup will be discussed in "Demonstration of Setup Potential".

\section{Setup-Validation Measurements}

To verify that the realized bending stage meets the design criteria mentioned in "Design Considerations and Criteria", validation measurements are performed, which are described in the following paragraphs.

\section{Moment Measurement Validation}

Firstly, the moment measurement and reproducibility of the testing device are validated by performing angularcontrolled bending tests on two equivalent monocrystalline silicon-wafer specimens (sample I and sample II), which are expected to behave linear-elastically. The thicknesses and widths of both specimens are $200 \mu \mathrm{m}, 2000 \mu \mathrm{m}$, respectively, and their lengths were optically measured to be $4905 \mu \mathrm{m}$, and $4834 \mu \mathrm{m}$, for sample I and sample II, respectively. The moment-curvature responses from the bending test are compared to analytic, pure bending solutions under plane stress and plane strain conditions. For the analytic calculations the cubic, orthotropic elastic parameters are chosen to be $E_{t}=166 \mathrm{GPa}$, and $v_{t z}=v_{z t}=0.064$ [19]. The results are shown in Fig. 7(a), in which the clearly observable linear-elastic responses of both silicon specimens are in good agreement with the analytic solutions, verifying the pure bending conditions in the test. Furthermore, the reproducibility of the bending test setup is demonstrated by the fact that the results of both tested specimens are equivalent.
Fig. 6 A right angle mirror is used to realize in-plane optical microscopic observation. The realization of the thickness perspective requires no mirror, since it already coincides with the vertical view-point of a typical optical microscope

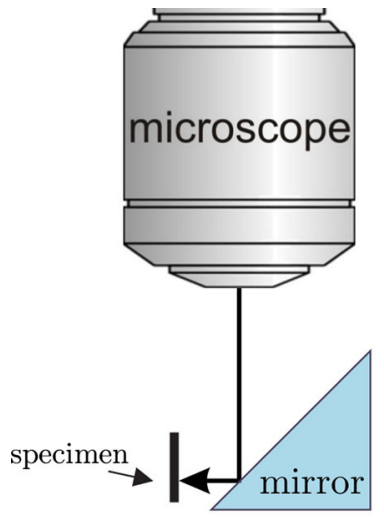

(a) mirror setup

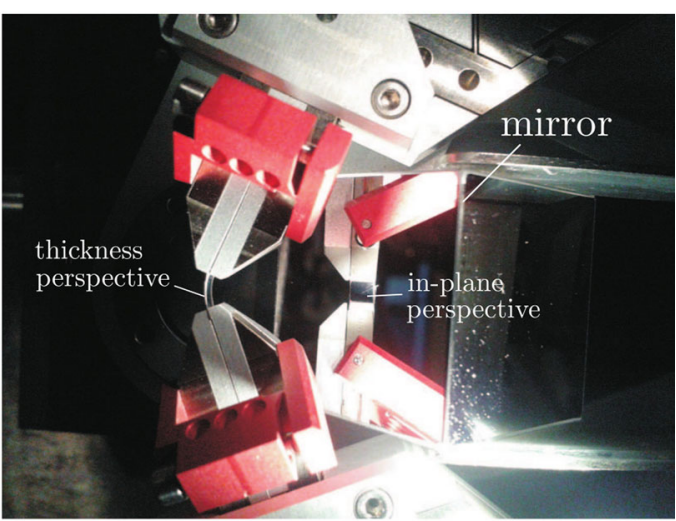

(b) microscope's view-point 


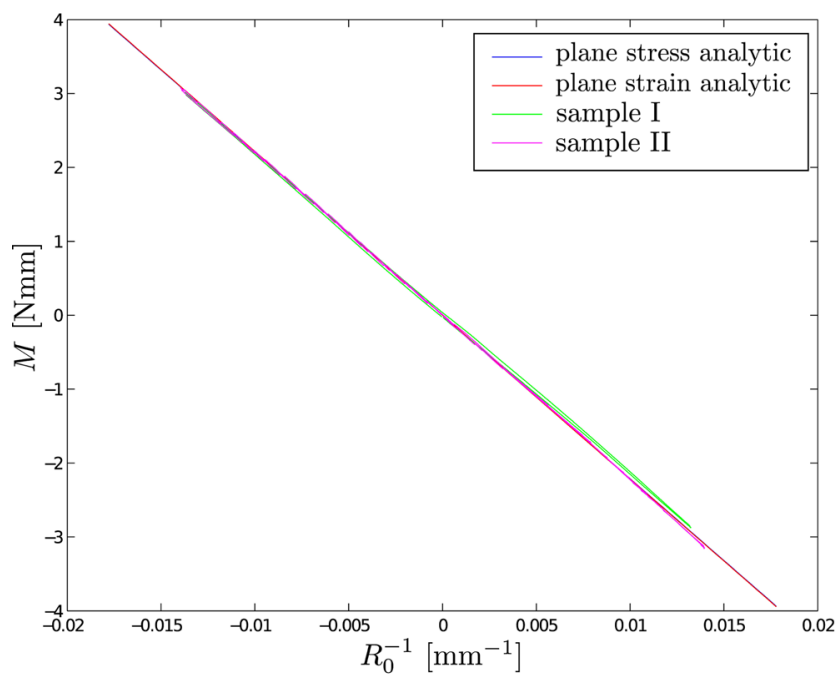

(a) moment-curvature

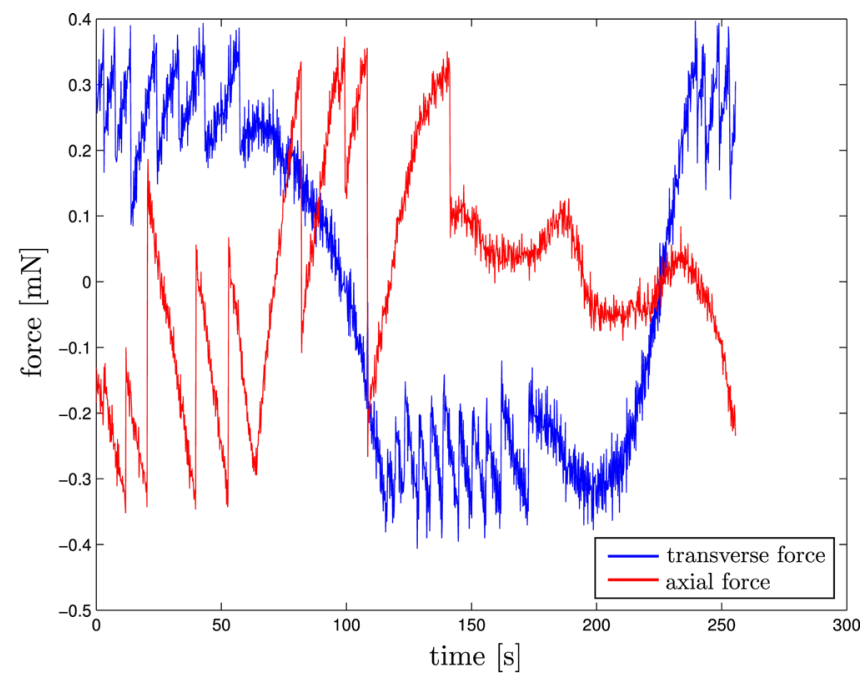

(b) axial and transverse forces

Fig. 7 moment-curvature plot (a) of two silicon specimens (sample I and II) being bent by the free bending stage. The results are compared to analytic solutions under plane stress and plane strain conditions. The axial and transverse forces (b) acting on the specimen during a test are actively controlled to stay below a predefined limit value

\section{Force Feedback Control Validation}

To ensure pure bending conditions, only momentapplication to the edges of the specimen must be guaranteed. To achieve this, any transverse and axial forces acting on the specimen's edges that cause parasitic moments should be eliminated by the active force feedback control system. Figure 7(b) shows the recorded transverse and axial forces acting on the specimen during a momentcontrolled experiment on silicon sample II. Their values never reach the predefined limit of equation (9), because of the active feedback control system. Hence, the parasitic moments resulting from these forces have values below the moment sensor's resolution, and therefore do not affect the measurement.

\section{Pure Bending Validation}

For a specimen that is homogeneous in its plane and is deformed according to a pure bending condition, the global strain can be directly determined by equation (1). The maximum tangential, tensile strain in the specimen becomes:

$\varepsilon_{\max }=\frac{h \theta}{2 L_{0}}$,

where $h$ is the thickness of the specimen, $L_{0}$ is its initial length, and $\theta$ the bending angle between the rotating clamps.

To verify whether a pure bending condition exist in the specimen, the corresponding global strain is compared to the maximum tangential, tensile strain from full-field measurements by Digital Image Correlation (DIC). This is done by an angular-controlled bending test on a $130 \mu \mathrm{m}$ thick polyethylene naphthalate (PEN) specimen. A speckle pattern is applied to the specimen, and the deformation is observed by optical microscopy and recorded by a CCDsensor. Global regularization is used in the DIC algorithm, meaning that instead of using local correlation of individual subsets, global shape functions are applied to describe the full displacement field. These shape functions are based on polynomials up to the third degree, which can accurately describe bending kinematics [20].

The comparison of the globally determined strain, by equation (11), and the maximum DIC-strain, is presented in Fig. 8 and are shown to be in good agreement within experimental uncertainty. The insets show the images of the speckle-patterned undeformed and bent PEN-specimen, used for DIC. The good agreement validates the global strain determination by bending kinematics, according to equation (11), and thereby verifies pure bending conditions in a homogeneous specimen. Furthermore, this result demonstrates the feasibility to accurately determine strain fields by DIC-analysis on experimental data from bending tests. Such ful7l-field measurements are in particular interesting for determining the position (shift) of the neutral axis of a specimen during bending, which is far from trivial, yet important for optimizing the design of flexible electronic systems.

\section{Cyclic Measurement Validation}

The following subsection concerns the validation of cyclic measurements with large angular rotations of the clamps. The specimen is a $130 \mu \mathrm{m}$ thick PEN specimen with an 


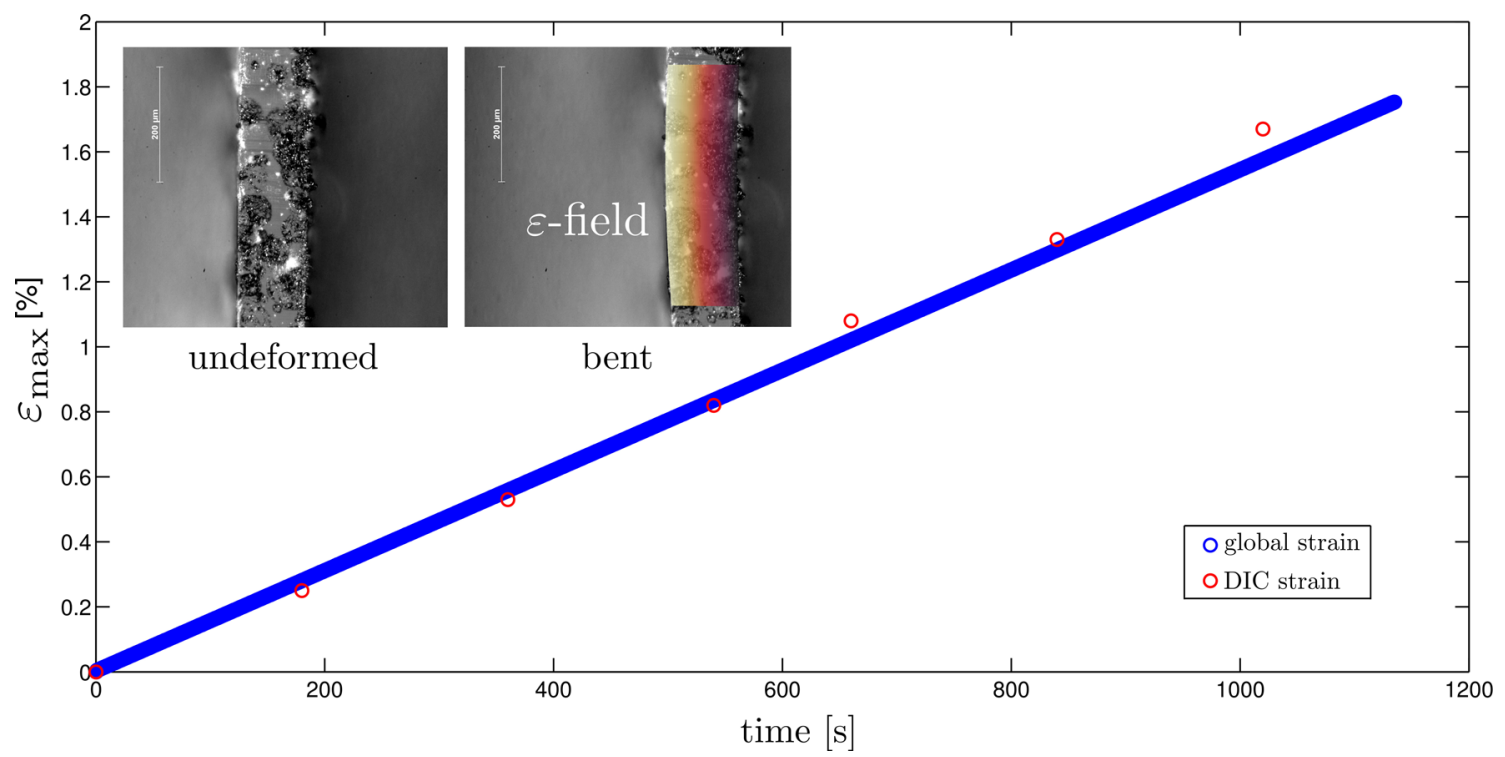

Fig. 8 comparison between the global maximum strain calculated from pure bending kinematics and the maximum strain determined from Digital Image Correlation, of which the strain-field is shown as an overlay in the image of the deformed bent specimen. Note that the maximum strain from DIC has been determined from a much smaller section of the specimen than the global maximum strain, which explains the larger statistical variation

optically measured length of $4938 \mu \mathrm{m}$, and a width of $2900 \mu \mathrm{m}$, which is subjected to cyclic, angular-controlled load application with a frequency of $\frac{1}{400} \mathrm{~Hz}$. The momentcurvature result is shown in Fig. 9. The maximum curvature corresponds to an angular rotation between the clamps of $\theta=108^{\circ}$, and a bending radius of $2.6 \mathrm{~mm}$. The observed hysteresis loop is not due to the test setup, but is caused by the nonlinear mechanical behavior of PEN, which was verified by a tensile test on the same type of specimen, of which the result is shown in the inset of Fig. 9.

This result demonstrates cyclic, large amplitude, load application, which allows for investigating multilayered material stacks in which different materials can be evaluated under tension and/or compression during one test. A cyclic test without hysteresis was already shown in Fig. 7(a) for a silicon specimen.
Fig. 9 moment-curvature ( $M$ $R_{0}^{-1}$ ) plot of a cyclic bending test on a PEN-specimen. The observed hysteresis loop is due to the nonlinear mechanical behavior of PEN, which was verified by a tensile test on a similar specimen (inset)

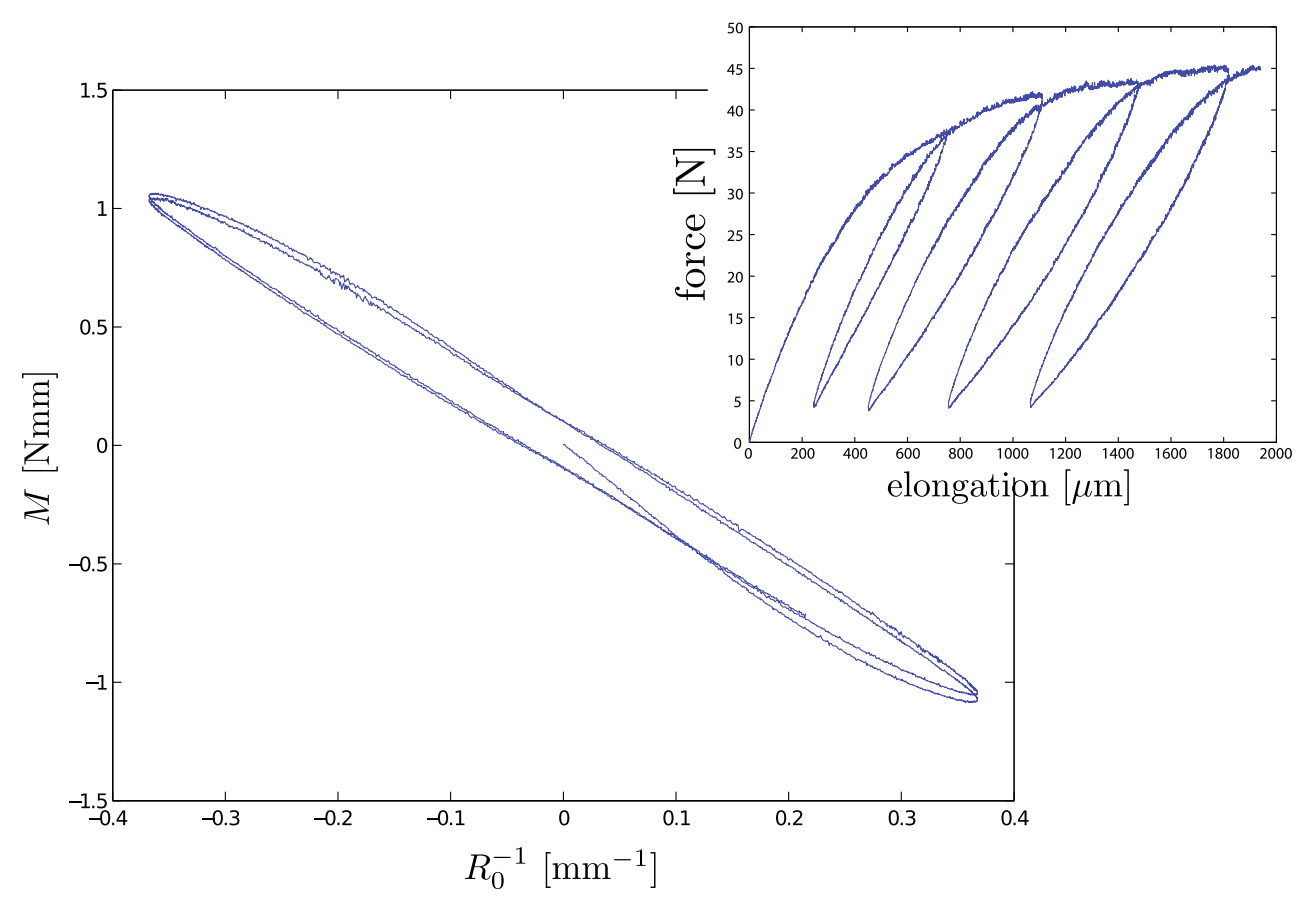


To conclude, it has been shown that the setup is capable of realizing pure bending conditions by accurate angular and moment-controlled load application to the edges of homogeneous specimens (silicon and PEN). Thereby, it was shown that any forces causing parasitic moments are successfully eliminated by active force feedback control.

\section{Demonstration of Setup Potential}

The following section describes experiments that demonstrate the potential of the free bending stage. To this end, insitu bending tests, making use of optical microscopy (OM) and Scanning Electron Microscopy (SEM), on multilayered samples were conducted. The tested material systems are especially interesting for the (flexible) microelectronics industry, in order to understand the different failure mechanisms. Note that these specific specimens are used for demonstration purposes, because of their versatility regarding the material composition (including polymers, ceramics, and metals), but the setup is capable of testing a wider range of materials and multilayered systems.

\section{Brittle Failure of a Barrier Coating on a Compliant Substrate}

Flexible electronics are produced by stacking layers of functional components onto a compliant substrate. To protect certain materials (e.g., electrodes and organic layers) against oxidation/hydroxidation, permeation barrier coatings must be incorporated, which are brittle and exhibit critical failure mechanisms, thereby jeopardizing the desired flexibility of the device. For the investigation of failure behavior of brittle barrier films used in flexible electronics, pure bending tests are conducted using the bending stage. Because of the contactless nature of load application in this test setup, microscopic investigatory techniques are easily applicable, which allows for detailed, microscopic investigation of failure mechanisms.

In-situ pure bending tests were conducted on bimaterial specimens, consisting of a $130 \mu \mathrm{m}$ thick substrate of PEN and a $600 \mathrm{~nm}$ thin film layer of $\mathrm{Si}_{3} \mathrm{~N}_{4}$ (used as a barrier coating in realistic flexible electronic), which is loaded either in tension or compression. Of each loading mode, a population of 13 specimens was tested, and the failure behavior was observed by optical microscopy (together with a rightangle mirror), viewing the in-plane fracturing of the brittle $\mathrm{Si}_{3} \mathrm{~N}_{4}$-film. This allows, among others, for recording the initial failure strain $\varepsilon_{i}$ at which the first crack is observed in a specimen during a bending test. Due to the stochastic nature of brittle fracturing, this behavior is commonly described by weakest link theories, such as Weibull statistics [21]. In order to obtain a value for the critical strain, and account for the statistical distribution of the failure strength, the experimental data from a multitude of pure bending tests on equivalent specimens is analyzed by Weibull statistics, in which the critical failure strain $\varepsilon_{c}$ is determined at 0.632 probability of the distribution.

Figure 10 shows a Weibull plot of the observed failure strains, together with images capturing the occurring cracks
Fig. 10 Weibull plots of $\mathrm{Si}_{3} \mathrm{~N}_{4}$-films bent in tension and compression. The horizontal orange line represents the 0.632 probability where the critical strain $\varepsilon_{c}$ is determined from the multiple tests on equivalent specimens. The optical micrographs (a) and (b) show tensile and compressive cracks in the plane of the film, which are believed to initiate near flaws in the thin film, as supported by evidence from SEM-investigation (c) (a)

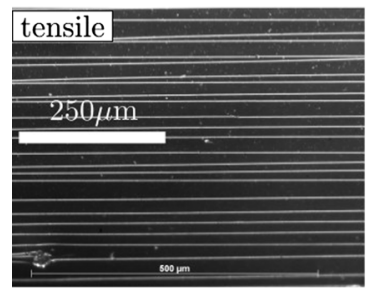

(b)

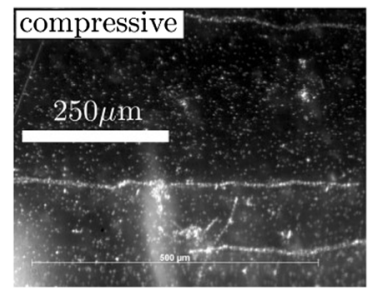

(c)

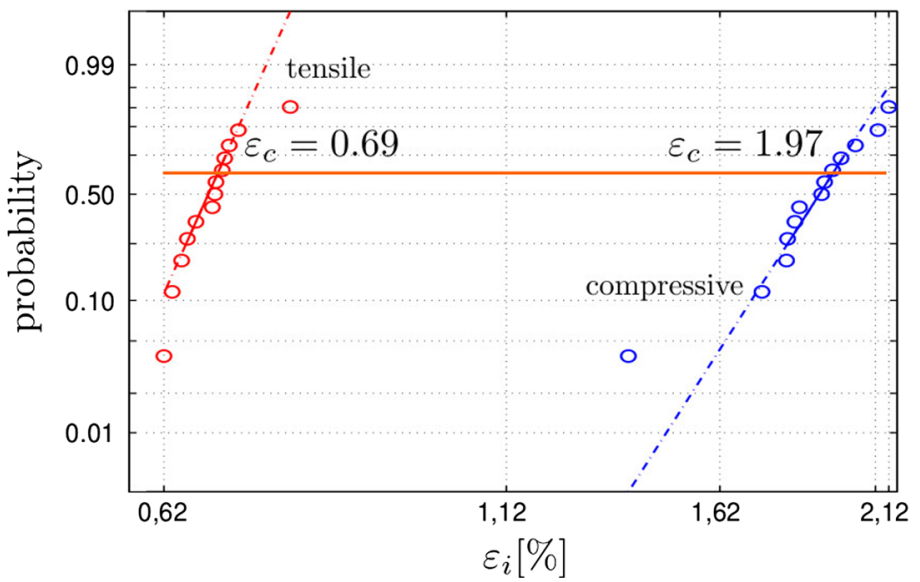




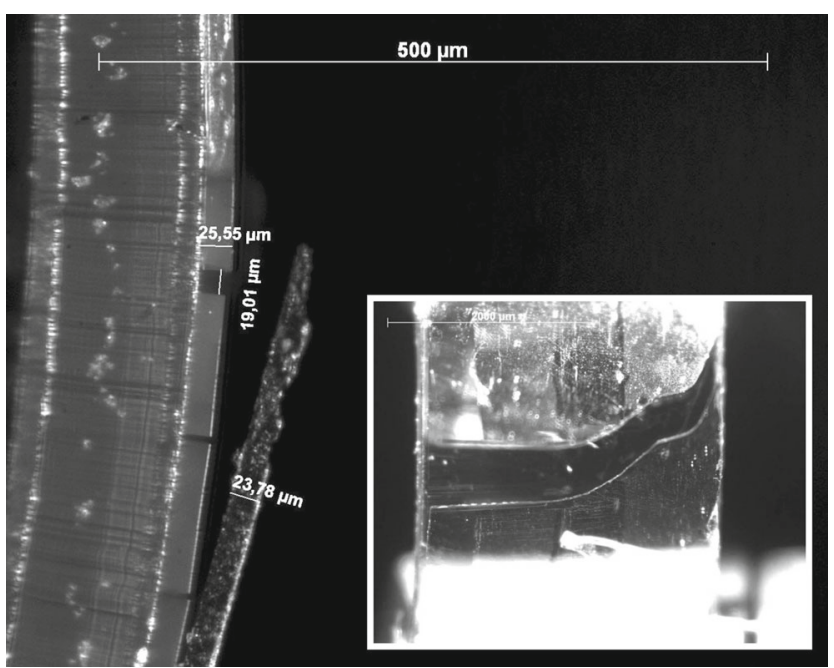

Fig. 11 OM-investigation of the material stack, viewing from both the thickness perspective and the in-plane perspective (inset), reveals that the outer layer fails at a strain of $\sim 2 \%$

during pure bending tests up to $80^{\circ}$ of angular clamp rotation, which corresponds to maximum absolute strain values in the film of $2.5 \%$. The critical strain in compression is three times higher than in tension, which may be due to energy-dissipating buckling delamination preceding fracturing in the compression load-case. Furthermore, for the same reason, the eventual cracks are less straight than the cracks observed in the tensile load-case. The cracks are believed to initiate near flaws or imperfections in the thin layer. Postexperiment SEM investigation of the cracked $\mathrm{Si}_{3} \mathrm{~N}_{4}$-film reveals flaw-like features near most of the observed cracks, of which a close-up example is also shown in Fig. 10(c).

\section{Failure of a Multilayered Material Stack}

Flexible electronics typically consist of multiple material layers stacked on top of one another. The stress distribution throughout the thickness of the stack is complex due to material mismatch. To study the failure behavior of these material stacks, in-situ bending tests with Optical Microscopy (OM) and Scanning Electron Microscopy (SEM) provide detailed information about the mechanical response to loading in tension and compression. The following example results show failure of $25 \mu \mathrm{m}$ thick brittle barrier coatings of multilayered flexible electronic specimens, with a PEN substrate, subjected to bending up to $80^{\circ}$ angular rotation of the clamps, in both tension and compression.

\section{Failure of layers in tension}

The outer layer fails at a critical tensile strain of $\sim 2 \%$, as was found by OM-observations viewing the thicknessdimension and in-plane dimension of the specimen, as shown in Fig. 11. Severe delamination of the outer layer follows immediately after first cracking, as seen in the images. Cracks are clearly visible in several layers, as seen in Fig. 11. However, the microscope's resolution $(\sim 500 \mathrm{~nm})$ is insufficient for the investigation of the detailed failure behavior of $150 \mathrm{~nm}$ thin $\mathrm{Si}_{3} \mathrm{~N}_{4}$-films that are sandwiched between the two thicker outer layers.

\section{Failure of layers in compression}

To realize investigation of thin films with thicknesses in the order of $100 \mathrm{~nm}$ or thinner, SEM-observation must be used. Figure 12 shows images resulting from an insitu pure bending test on the multilayered material stack, in which the barrier coatings, including the $150 \mathrm{~nm}$ thin $\mathrm{Si}_{3} \mathrm{~N}_{4}$-film, were loaded in compression. This allows for detailed investigation of complex failure modes, such as interface delamination and buckling, of several material layers.
Fig. 12 An in-situ, pure bending experiment using SEM results allowing for investigating delamination and buckling of different material layers with a resolution of $50 \mathrm{~nm}$

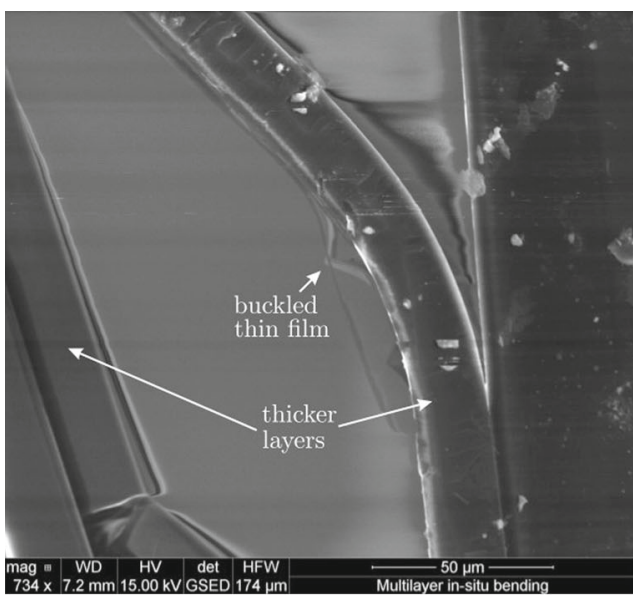

(a) failure of thin films

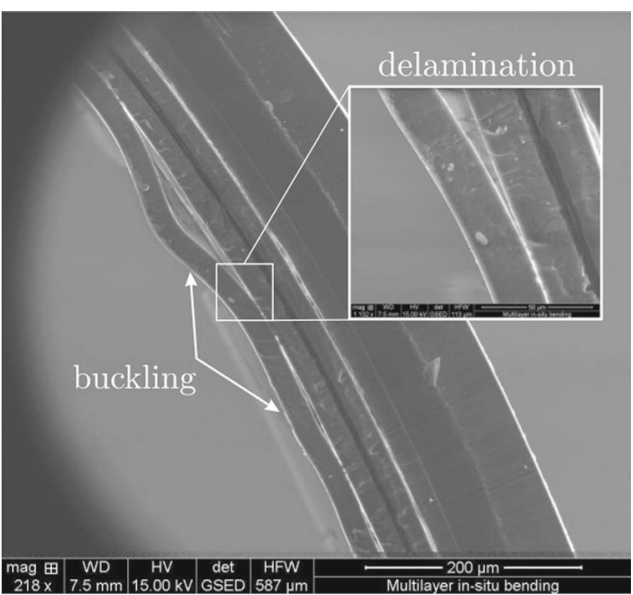

(b) failure of thicker layers 


\section{Conclusions and Recommendations}

Flexibility is a next-generation feature of electronics, which allows for a new range of electronic applications to be produced in a cost-effective roll-to-roll process. The realization of this type of electronics, however, remains to be challenging, and the first step in tackling these challenges, is to thoroughly investigate the utilized materials, in order to fully understand their (mechanical) failure behavior. To aid such research, a small-scale, autonomous, contactless, frictionless, large amplitude, cyclic, pure bending test setup was realized. The conceptual design was based on the pure bending kinematics of a homogeneous, orthotropic or isotropic, elastic specimen, and uses rotational hinges to transform an actuated linear translation into relative angular rotation of two gripper clamps up to $114^{\circ}$. To allow for testing other specimens with more heterogeneity and nonelastic behavior, an active force feedback control mechanism was incorporated to ensure pure moment-application to the edges of a small specimen $\left(4 \times(0-3) \times(0-0.4) \mathrm{mm}^{3}\right)$. Furthermore, to allow for in-situ testing, the bending stage was designed to fit inside the vacuum chamber of a SEM, and contains components that can withstand low vacuum conditions. Additionally, optical microscopy, in combination with a right-angle mirror, can be used to realize thickness and in-plane observation of the tested specimen.

Validation measurements, both moment-driven and angular-driven, were conducted to verify that the setup meets all the design criteria that were set up in "Design Considerations and Criteria". By reproducible bending tests on silicon specimens, of which results were compared to analytic calculations, accurate moment-measurement and realization of pure bending conditions in the specimen were verified. Furthermore, during the same tests it was verified that the setup was able to actively control the parasitic axial and transverse forces in the specimen so that they do not interfere with the moment application. It was shown that pure bending tests can be combined with DIC to determine strain-fields, which can be used to, e.g., accurately determine the neutral axis in a bent specimen. A DIC-analysis on a bent PEN-specimen validated the use of the global strain determination by pure bending kinematics. A similar PEN specimen was also used to validate large amplitude, cyclic measurements.

Realistic specimens, that are of particular interest to the flexible electronics industry were also tested. Brittle failure of thin films (with thicknesses in the order of $100 \mathrm{~nm}$ ) could be investigated by optical microscopy observing inplane fracturing of the coating material $\mathrm{Si}_{3} \mathrm{~N}_{4}$ on a compliant PEN-substrate. Both tensile and compression loading modes were triggered during bending tests, allowing for the determination of the corresponding critical failure strains, by using Weibull statistics. Furthermore, in-situ bending tests using OM and SEM were successfully performed to observe brittle fracturing, delamination, and buckling in samples from multilayered flexible displays with resolutions of $50 \mathrm{~nm}$. The application of the setup to such realistic specimens demonstrated the potential of the pure bending test to be used for quantitative investigation of complex material stacks.

Acknowledgments The authors would like to thank Marc van Maris and dr.ir. Nick Rosielle of the Mechanical Engineering department of the Eindhoven University of Technology (TU/e), dr. Piet Bouten of Philips Research Laboratories, and ir. Erwin Dekker of the Equipment \& Prototype Center of the TU/e for their contribution.

Open Access This article is distributed under the terms of the Creative Commons Attribution 4.0 International License (http:// creativecommons.org/licenses/by/4.0/), which permits unrestricted use, distribution, and reproduction in any medium, provided you give appropriate credit to the original author(s) and the source, provide a link to the Creative Commons license, and indicate if changes were made.

\section{References}

1. Nisato G, Mutsaers C, Buijk H, Duineveld P, Janssen E, de Goede J, Bouten P, Zuidema H (2004) Flexible PLED displays and related technologies. Mater Res Soc Symp Proc 814:177187

2. Leterrier Y, Bouten PCP, Jiang X (2001) Layer mechanics: experimental methods and models. European Delivery Report

3. Cotterell B, Chen Z (2000) Buckling and cracking of thin films on compliant substrates under compression. Int J Fract 104(2):169179

4. de Goede J, Bouten P, Médico L, Leterrier Y, Månson J-A, Nisato G (2005) Failure of brittle functional layers in flexible electronic devices. Mater Res Soc Symp Proc 854:190195

5. Bouten PCP, Van Gils MAJ (2005) Buckling failure of compressive loaded hard layers in flexible devices. Mater Res Soc Symp Proc 843:105-110

6. Bouten PCP (2005) On integrity of flexible displays. Fract Mech Ceram 14:597-608

7. Chen Z, Cotterell B, Wang W, Guenther E, Chua S-J (2001) A mechanical assessment of flexible optoelectronic devices. Thin Solid Films 394(12):201-205

8. Chen Z, Cotterell B, Wang W (2002) The fracture of brittle thin films on compliant substrates in flexible displays. Eng Fract Mech 69(5):597-603

9. Crawford GP (2005) Flexible flat panel displays. Wiley, New York

10. Boers SHA, Geers MGD, Kouznetsova VG (2010) Contactless and frictionless pure bending: principles, equipment and experimental opportunities. Proc Soc Exp Mech Inc 67:683693

11. Fenner RT (1989) Mechanics of solids. Blackwell Scientific Publications, Oxford

12. Yoshida F, Urabe M, Toropov VV (1998) Identification of material parameters in constitutive model for sheet metals from cyclic bending tests. Int J Mech Sci 40:237-249

13. Kolluri M, Hoefnagels JPM, van Dommelen JAW, Geers MGD (2011) An improved miniature mixed-mode delamination setup 
for in situ microscopic interface failure analyses. J Phys D Appl Phys 44(3):034005

14. Wiklund U, Bromark M, Larsson M, Hedenqvist P, Hogmark S (1997) Cracking resistance of thin hard coatings estimated by four-point bending. Surf Coat Technol 91:57-63

15. Frei H, Grathwohl G (1989) Development of a piezotranslatorbased bending device for in situ SEM investigations of high performance ceramics. J Phys E Sci Instrum 22(8):589593

16. Buchanan M, Provan JW, Gruzleski JE (1987) An apparatus for four-point bending fatigue testing of materials in a scanning electron microscope and its application to nodular cast iron. Metallography 20(2):125-143
17. Tasan CC, Hoefnagels JPM, Dekkers ECA, Geers MGD (2011) Multi-axial deformation setup for microscopic testing of sheet metal to fracture. Exp Mech 52(7):669-678

18. Hoefnagels JPM, Buizer CA, Geers MGD (2011) A miniaturized contactless pure-bending device for in-situ SEM failure analysis. Exp Appl Mech 6:587-596

19. Hopcroft MA, Nix WD, Kenny TW (2010) What is the Young's modulus of silicon? J Microelectromech Syst 19(2):229-238

20. Neggers J, Hoefnagels JPM, Hild F, Roux S, Geers MGD (2014) Direct stress-strain measurements from bulged membranes using topography image correlation. Exp Mech 54(5):717-727

21. Weibull W (1951) A statistical distribution function of wide applicability. J Appl Mech 18:293-234 